\title{
Angular momentum at the black hole threshold
}

\author{
Carsten Gundlach \\ Max-Planck-Institut für Gravitationsphysik (Albert-Einstein-Institut), Schlaatzweg 1, 14473 Potsdam, Germany
}

(Received 26 November 1997; published 20 May 1998)

\begin{abstract}
Near the black hole threshold in phase space, the black hole mass as a function of the initial data shows the "critical scaling" $M \simeq C\left(p-p_{*}\right)^{\gamma}$, where $p$ labels a family of initial data, $p_{*}$ is the value of $p$ at the threshold, and the critical exponent $\gamma$ is universal for a given matter model. The black hole charge $Q$ obeys a similar law. To complete the picture, we include the angular momentum as a perturbation. For the black hole angular momentum $\vec{L}$, we find the oscillating behavior $\vec{L} \simeq \operatorname{Re}\left[(\vec{A}+i \vec{B})\left(p-p_{*}\right)^{\mu+i \omega}\right]$. The assumptions of the calculation hold for $p=\rho / 3$ perfect fluid matter, and we calculate $\mu \simeq 0.799$ and $\omega \simeq 0.231$. [S0556-2821(98)50312-8]
\end{abstract}

PACS number(s): 04.70.Bw, 04.25.Dm, 04.40.Nr

\section{INTRODUCTION}

There has been much interest recently in critical phenomena at the black hole threshold. Take a smooth oneparameter family of smooth, asymptotically flat initial data for general relativity (with matter) that crosses the black hole threshold, and fine-tune the parameter, so as to get close to the black hole threshold. For many families, one can make arbitrarily small black holes this way. As discovered by Choptuik [1], the black hole mass near the threshold then scales as $M \simeq C\left(p-p_{*}\right)^{\gamma}$, where $p$ is the parameter of the initial data, $p_{*}$ is its value at the black hole threshold, and $\gamma$, the "critical exponent," is a universal constant common to all one-parameter families, while $C$ depends on the family.

In a nutshell, critical phenomena arise because general relativity, for a given matter model and symmetry, admits a "critical solution." By this we understand a strong-field solution that is an attractor of codimension one sitting on the black hole threshold, and whose basin of attraction is the black hole threshold. Perturbatively, this means that the critical solution has precisely one unstable mode, and that it decays into a black hole, or into flat space, depending on the sign of that mode. The critical solutions found so far are either static or periodic in time (type I), or continuously or discretely self-similar (type II). Both can arise in the same matter model $[2,3]$. Here we limit attention to type II, which gives rise to the power-law scaling of the mass. For a review of critical phenomena, see [4].

If a small amount of electric charge is present in the initial data, the black hole charge $Q$ obeys a similar power law as the mass $M$, with a new critical exponent $\delta[5,6]$. Black holes are completely characterized by their mass $M$, electric charge $Q$, and angular momentum $\vec{L}$. To complete the picture, here we consider initial data with small angular momentum. Our calculation assumes the existence, for a given matter model, of a spherically symmetric self-similar solution with exactly one unstable perturbation mode, including nonspherical perturbations.

Recently, we have shown for the first time that a spherically symmetric critical solution, namely that for perfect fluid matter with equation of state $p=\rho / 3$, has no unstable nonspherical perturbation modes [7]. The present abstract calculation therefore applies to this particular matter model, and gives to a quantitative prediction for it.

\section{NOTATION}

The calculation we give here is hardly more than an application of dimensional analysis. Therefore we set up a compact notation that hides all technical details specific to general relativity, in order to bring out the essential scaling argument. Let $g_{\mu \nu}$ be the spacetime metric in the coordinates $(\tau, x, \theta, \varphi)$, with $\theta$ and $\varphi$ the usual Euler angles, and let surfaces of constant $\tau$ be spacelike. If the conformal metric $\widetilde{g}_{\mu \nu} \equiv e^{2 \tau} g_{\mu \nu}$ is independent of $\tau$, then the spacetime is homothetic, or continuously self-similar (CSS). In this case, $\tau$ plays the double role of being the time coordinate and of being the negative logarithm of spacetime scale, while $x$ is dimensionless. A simple example of such a coordinate system is $\tau=-\ln (-t)$ and $x=r /(-t)$, where $t$ and $r$ are a standard choice of time and radial coordinate.

Let $Z(\tau, x, \theta, \varphi)$ stand for $\widetilde{g}_{\mu \nu}$ plus a suitable set of conformally rescaled matter variables. (For perfect fluid matter, for example, these are $\tilde{\rho}=e^{-2 \tau} \rho$ for the density, and $\tilde{u}_{\mu}$ $=e^{\tau} u_{\mu}$ for the four-velocity.) $Z(\tau, x, \theta, \varphi)$ then symbolizes a solution of the field equations, while $Z_{0}(x, \theta, \varphi)$ symbolizes Cauchy data. (To make this notion precise, we have to transform from $\widetilde{g}_{\mu \nu}$ to suitably rescaled Cauchy data $\widetilde{h}_{i j}$ and $\widetilde{K}_{i j}$.) Finally, let $Z(\tau, x, \theta, \varphi)=Z_{*}(x)$ stand for the critical solution, which is both spherically symmetric and CSS. (We overlook a trivial $\theta$ dependence, namely the factor $\sin ^{2} \theta$ in $g_{\varphi \varphi}$, for the sake of notation.)

For a solution that is a general perturbation of a selfsimilar, spherically symmetric background solution $Z_{*}(x)$, we can make the ansatz

$$
\begin{aligned}
Z(\tau, x, \theta, \varphi) \simeq & Z_{*}(x)+\sum_{l=0}^{\infty} \sum_{m=-l}^{l} \sum_{n=0}^{\infty} \\
& \times \operatorname{Re}\left[C_{l m n}(p) e^{\lambda_{l n} \tau} f_{l m n}(x, \theta \varphi)\right] .
\end{aligned}
$$

Because the background is spherically symmetric, we can decompose all linear perturbations into spherical harmonics labeled by $l$ and $m$. Because it is independent of $\tau$, the perturbation equations of motion separate, and the perturbations 
can be assumed to have a simple exponential dependence on $\tau$, where the separation constants $\lambda_{l m n}$ and the mode functions $f_{l m n}$ are complex. We assume here that the perturbation spectrum is countable and discrete, and label it by $n$ for each value of $l$ and $m$. Because $Z$ is real, the $\lambda_{l m n}$ and $f_{l m n}$ must be real or form complex conjugate pairs. Because of spherical symmetry, the $\lambda_{l m n}$ are in fact the same for all $m$, and are therefore labelled $\lambda_{l n}$. The $C_{l m n}$, also complex, are the free parameters of the generic perturbation. Here we want to consider one-parameter families of initial data labelled by a parameter $p$. The dependence of the solutions on $p$ is then a dependence of the free constants $C_{l m n}$ on $p$. The $f_{l m n}$ correspond to rescaled metric and matter perturbations in a suitable gauge, such as Regge-Wheeler gauge, or in a gaugeinvariant framework. We have addressed the technical details of gauge-fixing elsewhere [7].

\section{REVIEW OF MASS SCALING}

We now consider the time evolution of initial data that start close to the spherically symmetric self-similar critical solution, so that the expansion (1) applies initially. The critical solution contracts to a curvature singularity at $\tau=\infty$. As the singularity is approached from the past, with $\tau$ increasing, a perturbation grows (decays) if the real part of its $\lambda$ is positive (negative). By definition, a critical solution has precisely one unstable mode. Being unique, this unstable mode must be spherically symmetric because all higher values of $l$ are $(2 l+1)$-fold degenerate and its $\lambda$ must be real. We label its mode function $f_{00 *}$ and its eigenvalue $\lambda_{0 *}$.

Close enough to the singularity, we can neglect all stable perturbation modes. The unstable perturbation keeps growing, however, and eventually the solution deviates nonlinearly from the critical solution. The solution eventually forms a black hole for $p>p_{*}$, but disperses for $p<p_{*}$. As $p \rightarrow p_{*}$ from either side, the solution approximates the critical solution to ever smaller scales, that is to ever larger $\tau$. This means that $C_{00 *}(p)$ must vanish precisely at $p=p_{*}$. Linearizing $C_{00 *}$ around $p=p_{*}$, and neglecting all decaying perturbations, we have

$$
Z \simeq Z_{*}(x)+\frac{\partial C_{00 *}}{\partial p}\left(p_{*}\right)\left(p-p_{*}\right) e^{\lambda_{0 *} \tau} f_{00 *}(x) .
$$

For $p>p_{*}$, we define $\tau_{p}$ as a function of $p$ by

$$
\frac{\partial C_{00 *}}{\partial p}\left(p_{*}\right)\left(p-p_{*}\right) e^{\lambda_{0 *} \tau_{p}} \equiv \epsilon,
$$

where $\epsilon$ is an arbitrary small constant.

We now consider the Cauchy data $Z_{0}$ given by

$$
Z\left(\tau_{p}, x, \theta, \varphi\right) \simeq Z_{*}(x)+\epsilon f_{00 *}(x) \equiv Z_{0}(x) .
$$

We have chosen $\epsilon$ small enough that the growing mode has not yet gone nonlinear (its exact value does not matter). For larger $\tau$, the linearized ansatz (1) breaks down, and we know only that the solution forms a black hole and settles down to Schwarzschild. We have defined $\tau_{p}$ so that the rescaled variables $Z$ are independent of $p$ in the Cauchy data $Z_{0}$. The field equations themselves are scale-invariant (or asymptotically scale-invariant on small scales). As a result, there is only one scale, $e^{-\tau_{p}}$, in the problem, and therefore the black hole mass must be proportional to it. We have

$$
M \simeq M_{0} e^{-\tau_{p}} \simeq C\left(p-p_{*}\right)^{\gamma}, \quad \gamma=\frac{1}{\lambda_{0 *}},
$$

where $M_{0}$ is a universal constant, and $C$ is a familydependent constant absorbing $\partial C_{00 *} / \partial p\left(p_{*}\right)$. This derivation of the critical scaling of the black hole mass has been given before [8-11]. Collapse simulations show that the critical solution is an attractor, and the mass scaling (5) holds, even beyond the strict applicability of the perturbation ansatz (1).

\section{ANGULAR MOMENTUM SCALING}

Now we generalize the above discussion to initial data with nonvanishing angular momentum. Our discussion holds strictly only for initial data which are almost spherically symmetric, so that deviations from spherical symmetry can be treated as linear perturbations throughout the evolution. In particular, this means that our results hold only for black holes whose angular momentum is much smaller than their mass, so that the final Kerr solution can be treated as a perturbation of Schwarzschild. If the critical solution turned out to be an attractor beyond the linear regime also for nonspherical spherical initial data, our discussion and results could then hold also for larger deviations from spherical symmetry.

A Kerr black hole metric with small angular momentum $\left(L \ll M^{2}\right)$ can be written as a Schwarzschild metric with a linear perturbation proportional to $L$. In fact, the gauge can be chosen so that the shift component of the metric is

$$
g_{t \varphi}=2 \frac{L}{r} \sin \theta \frac{\partial}{\partial \theta} P_{1}+O\left(L^{3} / M^{6}\right)
$$

where $P_{1}$ is a Legendre polynomial, while all other metric coefficients are those of the Schwarzschild metric in the usual Schwarzschild coordinates, plus perturbations of $O\left(L^{2} / M^{4}\right)$ [12]. This result can be verified immediately by expanding the Kerr solution in Boyer-Lindquist coordinates around $L=0$. The only perturbation to leading order in $L$ therefore has $l=1$ angular dependence and odd parity in the Regge-Wheeler notation. Here the orientation of the coordinate axes was chosen so that the perturbation is purely $m$ $=0$. For a general orientation of $\vec{L}$ relative to the coordinate axes, $m=-1,0,1$ will all appear. As a subdominant effect, we therefore include the odd $l=1$ perturbations in the data $Z_{0}$ even though they are subdominant, with the aim of learning something about the behavior of the black hole angular momentum near criticality.

We only keep the dominant odd $l=1$ mode, that is the one with the largest (least negative) real part of $\lambda$, and denote it by $\lambda_{1 *}$. Generally, $\lambda_{1 *}$ will not be real, but part of a complex conjugate pair. $l=1$ is also threefold degenerate, with mode functions $f_{1 m *}$ for $m=-1,0,1$, or the three directions in space. Keeping these modes results in a small perturbation of the initial data $Z_{0}$ :

$$
Z\left(\tau_{p}, x, \theta, \varphi\right) \simeq Z_{0}(x)+e^{\operatorname{Re} \lambda_{1 *} \tau_{p}} \delta Z_{0}\left(\tau_{p}, x, \theta, \varphi\right),
$$


where

$$
\delta Z_{0}=\operatorname{Re} \sum_{m=-1}^{1} C_{1 m *}\left(p_{*}\right) e^{i \operatorname{Im} \lambda_{1 *} \tau_{p}} f_{1 m *}(x, \theta, \varphi)
$$

is periodic in $\tau_{p}$. As long as its amplitude $e^{\operatorname{Re} \lambda_{1 *} \tau_{p}}$ is small enough, the perturbation $\delta Z_{0}$ evolves as a linear perturbation of the spacetime generated by the spherically symmetric data $Z_{0}$ all way until the solution has settled down to Schwarzschild with a small odd-parity $l=1$ perturbation that turns it into a slowly rotating Kerr solution. By the black hole nohair theorem, no other perturbations (except an electric charge of the black hole) can survive in the final state. This justifies our considering odd $l=1$ perturbations, but neglecting $l>1$ and even perturbations altogether. In the linear approximation, the black hole angular momentum $L$ must, on average in $\tau_{p}$, be proportional to $e^{\operatorname{Re} \lambda_{1 *} \tau_{p}}$. Because this is a dimensionless number, we must have

$$
\frac{L}{M^{2}} \propto e^{\operatorname{Re} \lambda_{1 *} \tau_{p}}
$$

for the overall scaling of $L$. But there is an interesting modulation due to the fact that $\delta Z_{0}$ depends periodically on $\tau_{p}$, as we shall see now.

By linearity, the presence of a perturbation $\epsilon \operatorname{Re} f_{1 m *}$, for some small $\epsilon$, in the initial data $Z_{0}$ must give a rise to a small angular momentum component $L_{z} / M^{2}=\epsilon A$ in the final black hole, while $\epsilon \operatorname{Im} f_{1 m *}$ gives rise to $L_{z} / M^{2}=\epsilon B$. Similar proportionalities apply to $\operatorname{Re} f_{1 m *}$ and $\operatorname{Im} f_{1 m *}$ for $m= \pm 1$, and the $x$ and $y$ components of the black hole angular momentum. We absorb the universal, but unknown proportionality factors and the family-dependent constants $C_{1 m *}$ together into six new constants $\vec{A}$ and $\vec{B}$. Putting all the factors together, we obtain the following final result for the angular momentum vector $\vec{L}$ of the black hole as a function of the initial data, for initial data near the black hole threshold and near spherical symmetry:

$$
\vec{L}=\operatorname{Re}\left[(\vec{A}+i \vec{B})\left(p-p_{*}\right)^{\mu+i \omega}\right]
$$

where

$$
\mu=\frac{2+\operatorname{Re} \lambda_{1 *}}{-\lambda_{0 *}}, \quad \omega=\frac{\operatorname{Im} \lambda_{1 *}}{-\lambda_{0 *}},
$$

and $\vec{A}$ and $\vec{B}$ are six family-dependent constants. Note that we always have $\mu>2 \gamma$ because $\operatorname{Re} \lambda_{1 *}<0$ by the assumptions of this calculation.

This is our main result. As $p \rightarrow p_{*}$ from above in the initial data, the angular momentum vector $\vec{L}$ of the final black hole rotates in space as it decreases. It describes a rapidly shrinking ellipse of size $\left(p-p_{*}\right)^{\mu}$. This behavior formally resembles a damped isotropic three-dimensional harmonic oscillator, with $-\ln \left(p-p_{*}\right)$ playing the role of "time," $\omega$ the frequency, and $\mu$ the damping. Of course, $p$ $-p_{*}$ is not in any sense a time, but a measure of the distance, in phase space, of the initial data set labeled by $p$ from the black hole threshold.

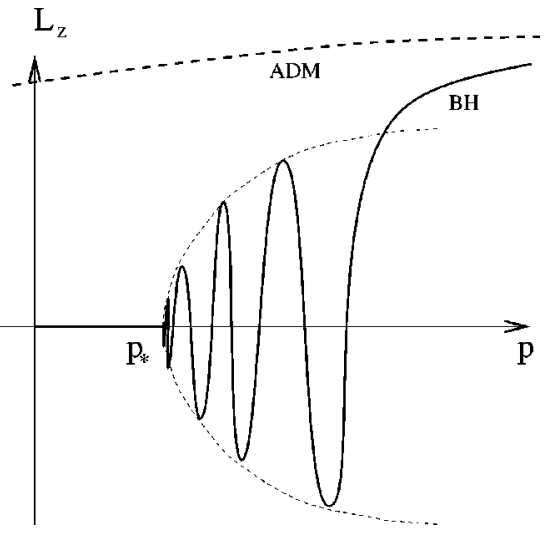

FIG. 1. Schematic plot of the ADM angular momentum (thick dashed line) and the angular momentum of the black hole (thick full line) in axisymmetric collapse in a one-parameter family of axisymmetric data crossing the black hole threshold. $p$ is the parameter of the initial data and $p_{*}$ is its value at the black hole threshold. The envelope (thin dashed line) is the overall power law. There is an infinite number of oscillations as $p \rightarrow p_{*}$ from above.

\section{THE AXISYMMETRIC CASE}

The result (10) is particularly surprising if we consider a family of strictly axisymmetric initial data. Then $\vec{L}$ can only point along the symmetry axis, and the ellipse degenerates into a line:

$$
L=L_{z}=\left(p-p_{*}\right)^{\mu} A \cos \left[\omega \ln \left(p-p_{*}\right)+c\right],
$$

where $A$ and $c$ are two family-dependent constants. If the family of axisymmetric initial data has angular momentum that does not vanish at $p=p_{*}$, then the angular momentum of the black hole, as a function of the initial data, alternates between parallel and antiparallel to the angular momentum of the initial data. The simple power-law scaling $L_{z}=(p$ $\left.-p_{*}\right)^{\mu}$ one might naively expect would be recovered only if $\lambda_{1 *}$ was purely real. The oscillating behavior is sketched in Fig. 1. It is perhaps as dramatic an illustration of the "forgetting" of initial data in critical collapse as the universality of the critical solution itself.

\section{DISCRETE SELF-SIMILARITY}

Here we have considered the effects of a critical solution that is CSS, both for simplicity of notation and because the one case that our calculation is actually known to apply to (the perfect fluid) is CSS. It is straightforward, however, to generalize our result for the angular momentum scaling to hypothetical critical solutions that are DSS instead of CSS. The periodic dependence of the angular momentum in CSS critical collapse is a simple cosine, because it derives from a time-dependence $\exp \lambda_{1 *} \tau$ of the leading $l=1$ odd perturbation, with $\lambda_{1 *}$ complex. In DSS critical collapse, the background critical solution itself is periodic in $\tau$, in a nontrivial way, with a period $\Delta$. Therefore, one would expect both kinds of periodicity to arise in $\vec{L}$. The calculation bearing this out is a straightforward generalization of the one for the CSS case, and here we only give its result. It is 


$$
\vec{L}=\operatorname{Re}\left\{\mathcal{M}\left[\ln \left(p-p_{*}\right)+c\right](\vec{A}+i \vec{B})\left(p-p_{*}\right)^{\mu+i \omega}\right\},
$$

where the only difference is the presence of the complex 3 $\times 3$ matrix $\mathcal{M}$. It is universal and can in principle be calculated from the mode functions $f_{1 m *}$. In the CSS case it can be assumed to be 1 . It is periodic in its argument $\ln \left(p-p_{*}\right)$ with period $\Delta / \gamma . c$ is a family-dependent constant. The role of $\mathcal{M}$ is similar to that of the fine structure of the black hole mass scaling in the DSS case $[11,13]$ (where the same parameter $c$ also appears).

\section{PREDICTIONS FOR THE $p=\rho / 3$ PERFECT FLUID}

We conclude this paper with a quantitative prediction, based on numerical work reported elsewhere [7]. The previ- ously known critical solution (in spherical symmetry) for the perfect fluid with equation of state $p=\rho / 3$ [14] was perturbed, with the result that, at least perturbatively around spherical symmetry, it remains the critical solution in the full phase space. The assumptions of the present paper therefore apply to this matter model. The values $\lambda_{0 *} \simeq 2.785$ and $\lambda_{1 *} \simeq-0.226 \pm 0.644 i$ were found numerically. The value of $\lambda_{0 *}$ gives rise to a critical exponent for the black hole mass $\gamma=1 / \lambda_{0 *} \simeq 0.359$, in agreement with the value 0.3558 given previously $[8,9]$. Therefore, we predict, for this matter model, for initial data near spherical symmetry and near the black hole threshold, the oscillatory behavior (10), with $\mu$ $\simeq 0.799$ and $\omega \simeq 0.231$. For axisymmetric critical collapse in particular (which may be simpler to investigate numerically than the general case) this reduces to the scaling (12) with the same $\mu$ and $\omega$ (as $\lambda_{1 *}$ is not purely real).
[1] M. W. Choptuik, Phys. Rev. Lett. 70, 9 (1993).

[2] M. W. Choptuik, T. Chmaj, and P. Bizon, Phys. Rev. Lett. 77, 424 (1996).

[3] P. R. Brady, C. M. Chambers, and S. M. C. V. Gonçalves, Phys. Rev. D 56, 6057 (1997).

[4] C. Gundlach, Adv. Theor. Math. Phys. 2, 1 (1998).

[5] C. Gundlach and J. M. Martín-García, Phys. Rev. D 54, 7353 (1996).

[6] S. Hod and T. Piran, Phys. Rev. D 55, 3485 (1997).

[7] C. Gundlach, Phys. Rev. D (to be published), gr-qc/9710066.
[8] T. Koike, T. Hara, and S. Adachi, Phys. Rev. Lett. 74, 5170 (1995).

[9] D. Maison, Phys. Lett. B 366, 82 (1996).

[10] E. W. Hirschmann and D. M. Eardley, Phys. Rev. D 52, 5850 (1995).

[11] C. Gundlach, Phys. Rev. D 55, 695 (1997).

[12] R. J. Gleiser, C. O. Nicasio, R. H. Price, and J. Pullin, Phys. Rev. D 57, 3401 (1998).

[13] S. Hod and T. Piran, Phys. Rev. D 55, 440 (1997).

[14] C. R. Evans and J. S. Coleman, Phys. Rev. Lett. 72, 1782 (1994). 